not only professional bone-resorbing cells but also directly involved in controlling inflammatory responses. Depending on the pathophysiological context, OC can polarize the immune response towards tolerance or inflammation. Aiming at identifying key regulators of inflammatory OC functions, we have defined a miRNA-based signature, which includes miR-342-3p. MiRNAs are key regulators of gene expression that control cellular processes, including osteoclastogenesis, and few miRNAs have been described in the differentiation of myeloid precursors into mature OC.

Objectives To study the role of miR-342-3p in inflammatory OC.

Methods OC were derived from the murine monocyte RAW264.7 cells. The expression levels of miR-342-3p and OC-specific genes were monitored by qRT-PCR. RAW264.7 cells were transfected with either miR-342-3p mimics, neutralizing molecules or control miRNAs. Cell survival and proliferation were assessed at 48 hour after RANKL incubation using quantification of the caspase3-7 activity, ATP production and BrDU incorporation. Motility of OC precursors was monitored using time-laps during the course of OC differentiation. The $\mathrm{K} / \mathrm{BxN}$ serum-transfer arthritis (STA) model was performed in 8 weeks old $\mathrm{C} 57 \mathrm{BL} / 6$ males and bone marrow was flushed. Primary OC were generated from either total bone marrow or sorted $\mathrm{CD}_{11} \mathrm{~b}^{+}$and $\mathrm{CD} 11 \mathrm{c}^{+}$cell subsets of healthy and arthritic mice.

Results The expression of miR-342-3p was transiently up-regulated in the early phase of OC generation and was down-regulated after 24-48 hour in OC precursors. While pre-miR-342$3 p$ promoted the motility of RAW264.7 cells, anti-miR-342-3p inhibited all motility parameters recorded $(p<0.0001$, ANOVA test). Anti-miR-342-3p reduced the proliferation $(p<0.01)$ and cell survival of OC precursors through a pro-apoptotic effect, as assessed by increased caspase $3-7$ activity $(p<0.01)$. Overall, miR-342-3p neutralization in OC precursors reduced OC numbers $(p<0.001)$ compared to the pre-miR-342-3p condition. In primary cells, miR-342-3p was up-regulated in bone marrowderived mature OC from arthritic mice compared to healthy controls ( $p=0.03$; STA $n=5 /$ group).

Conclusions Our data suggest that miR-342-3p promotes the early phase of osteoclastogenesis by enhancing the cell survival and motility of OC precursors. The up-regulation of miR-342$3 \mathrm{p}$ in OC isolated from arthritic mice may reflect the increased osteoclastogenic potential of inflammatory precursors in arthritis.

Disclosure of Interest None declared.

\section{P067 TOFACITINIB IMPAIRS MONOCYTE-DERIVED DENDRITIC CELL DIFFERENTIATION IN RHEUMATOID ARTHRITISAND PSORIATIC ARTHRITIS}

${ }^{1}$ V Marzaioli*, ${ }^{1} \mathrm{M}$ Canavan, ${ }^{1} \mathrm{~A}$ Floudas, ${ }^{1} \mathrm{~S}$ Wade, ${ }^{2} \mathrm{C}$ Low, ${ }^{2} \mathrm{DJ}$ Veale, ${ }^{1} \mathrm{U}$ Fearon. ${ }^{1} \mathrm{TBSI}$, Molecular Rheumatology, Trinity College Dublin; ${ }^{2}$ Centre for Arthritis and Rheumatic Diseases, St Vincent's University Hospital, UCD, Dublin, Ireland

\subsection{6/annrheumdis-2018-EWRR2019.56}

Career situation of first and presenting author Post-doctoral fellow.

Introduction Tofacitinib is an oral Janus kinase inhibitor, recently approved for the treatment of rheumatoid arthritis (RA) and psoriatic arthritis (PsA). Although its mechanism of action has been explored in circulating cells, including neutrophils and lymphocyte, its effect on dendritic cells development and function remains still to be elucidated. Monocyte-derived dendritic cells (Mo-DC) are a subset of inflammatory DC derived from circulating monocytes with a key role in inflammation and infection.

Objectives The aim of this project is to evaluate the effect of Tofacitinib on inflammatory Mo-DC differentiation from RA and PsA patients, an important step in innate immunity.

Methods Monocytes were isolated from blood of healthy donor (HC), RA and PsA patients by magnetic separation and differentiated in the presence of GM-CSF/IL-4 cocktail for 7 days. To evaluate the effect of Tofacitinib on Mo-DC differentiation, monocyte were pre-treated with $1 \mu \mathrm{M}$ Tofacitinib (or DMSO as control). CD209 (immature DC marker) was evaluated by flow cytometry in the $\mathrm{CD}_{11} \mathrm{c}^{+}$population. Non-specific macropinocytosis (using Lucifer Yellow) and receptormediated endocytosis (using $\mathrm{DQ}^{\mathrm{TM}}$ Ovalbumin) were investigated by flow cytometry. The effect of Tofacitinib on NADPH oxidases (NOX) 5 and 2 expression, known players in MoDC differentiation, was evaluated by Western blot analysis. Finally, the frequency of $\mathrm{CD} 209^{+}$cells and their chemokine receptor expression (CXCR3/4/5 and CCR6/7) were evaluated by flow cytometry in peripheral blood (PBMC), synovial fluid (SFMC) mononuclear cells and synovial tissue cell suspensions from RA and PsA patients.

Results Pre-treatment of Mo-DC with Tofacitinib inhibited Mo-DC differentiation in RA and PsA patients, as evident by reduced CD209 marker expression. The decreased ability of monocytes to differentiate into DC in the presence of Tofacitinib was translated into a functional impairment of endocytic ability, in particular in PsA patients, as observed by the decreased uptake of both $\mathrm{DQ}^{\mathrm{TM}}$ Ovalbumin and Lucifer Yellow. In addition, Tofacitinib decreased NOX5 and increased NOX2 protein expression in Mo-DC of PsA and RA Mo-DC, altering the NOX2/NOX5 balance. Finally, we identified $\mathrm{CD} 09^{+}$cells in the periphery of RA and PsA patients, which were enriched in SFMC and synovial tissue cell suspension, and presented with an increased expression of CCR7 and CXCR3/5.

Conclusions Together, these observations suggest a novel mechanism of action of Tofacitinib in RA and PsA, by inhibiting Mo-DC development, which may alter migration of DC to the joint and subsequent activation of the immune response.

Disclosure of Interest V. Marzaioli Grant/research support from: Pfizer, M. Canavan: None declared, A. Floudas : None declared, S. Wade : None declared, C. Low : None declared, D. Veale: None declared, U. Fearon Grant/research support from: Pfizer.

\section{P068 RHEUMATOID ARTHRITIS PERIPHERAL CD14+ MONOCYTES ARE HYPER-INFLAMMATORY, HYPER- GLYCOLYTIC AND RETAIN A MEMORY BIAS TOWARD M1 MACROPHAGES}

${ }^{1} \mathrm{~T}$ Mcgarry*, 'M Hanlon, ${ }^{1} \mathrm{U}$ Fearon, ${ }^{2} \mathrm{D}$ J Veale. ${ }^{1}$ Clinical Medicine, Trinity Biomedical Sciences Institute; ${ }^{2}$ Centre for Arthritis and Rheumatic Diseases, St. Vincent's University Hospital, Dublin, Ireland

\subsection{6/annrheumdis-2018-EWRR2019.57}

Career situation of first and presenting author Post-doctoral fellow.

Introduction Myeloid cells with a monocyte/macrophages phenotype are present in large numbers in the rheumatoid arthritis (RA) joint, significantly contributing to disease. 